MODELO DE REFERÊNCIA PARA TRANSFERÊNCIA E ESTOCAGEM DE ALTO DESEMPENHO

Dissertação apresentada ao Programa de Pósgraduação em Engenharia Industrial - PUC-Rio. como requisito parcial para obtenção do título de Mestre em Engenharia de Produção.

Orientador: Prof. Dr. Madiagne Diallo 


\section{MODELO DE REFERÊNCIA PARA TRANSFERÊNCIA E ESTOCAGEM DE ALTO DESEMPENHO}

Dissertação apresentada ao Programa de Pósgraduação em Engenharia Industrial - PUC-Rio. como requisito parcial para obtenção do título de Mestre em Engenharia de Produção.

Prof. Dr. Madiagne Diallo

Orientador

Departamento de Engenharia Industrial - PUC-Rio

Prof. Dr. Nélio Domingues Pizzolato Departamento de Engenharia Industrial - PUC-Rio

Dr. Ricardo Vitor Jacomino da Cunha Vasconcellos

Petrobras

Prof. Dr. José Eugênio Leal Coordenador Setorial do Centro Técnico Científico - PUC-Rio 
Todos os direitos reservados. É proibida a reprodução total ou parcial do trabalho sem autorização da universidade, da autora e do orientador.

\section{Juliana Guimarães Cardoso de Mello}

Graduou-se em Administração de Empresas pela Universidade Federal de Juiz de Fora - UFJF. É consultora sênior de negócios de uma multinacional onde atua profissionalmente desde 2004.

Ficha Catalográfica

Mello, Juliana Guimarães Cardoso de

Modelo de referência para transferência e estocagem de alto desempenho / Juliana Guimarães Cardoso de Mello ; orientador: Madiagne Diallo. - 2008.

118 f. : il. ; $30 \mathrm{~cm}$

Dissertação (Mestrado em Engenharia Industrial)-Pontifícia Universidade Católica do Rio de Janeiro, Rio de Janeiro, 2008.

Inclui bibliografia

1. Engenharia industrial - Teses. 2. Modelo de referência. 3. Petróleo. 4. Refinaria. 5. Transferência. 6. Estocagem. I. Diallo, Madiagne. II. Pontifícia Universidade Católica do Rio de Janeiro. Departamento de Engenharia Industrial. III. Título. 


\section{Dedicatória}

Para você Fábio “que esperou minha demora, suportou minha ausência, que aceitou calar-se perante minha ira, mas não se privou de falar diante de meus erros. Que fingiu ausentar-se quando eu desejava a solidão e se fez presença alegre nos momentos difíceis. Que soube ser terra quando meus sonhos eram altos demais, e céu quando eu perdia as perspectivas. Que interpretou meus olhos todo o tempo e foi cúmplice em cada atitude. A você, que dividiu comigo alegria, choro, sonho e paixão.” 


\section{AGRADECIMENTOS}

A Deus pela paz e luz;

Ao meu marido Fábio que com amor, paciência, carinho e sabedoria me apoiou durante essa jornada;

Aos meus pais que me educaram e permitiram que eu chegasse até aqui para mais uma conquista;

À minha madrinha Lê, suporte essencial em minha vida no Rio de Janeiro;

À Cláudia Teti, perfeita secretária, sempre disponível e disposta a resolver todos os obstáculos surgidos durante o mestrado; 
Ao Dr. Ricardo Vasconcellos pela tranquilidade e apoio durante a defesa;

Ao Mário Jorge pelo apoio efetuado durante o trabalho;

À PUC ao suporte acadêmico e auxílio financeiro. 


\section{Resumo}

Mello, Juliana; Diallo, Madiagne. Modelo de Referência para Transferência e Estocagem de Alto Desempenho. Rio de Janeiro, 2008. 118 p. Dissertação de Mestrado - Departamento de Engenharia Industrial. Pontifícia Universidade Católica do Rio de Janeiro.

Este trabalho tem como objetivo realizar um estudo de modelagem dos processos e identificação das melhores práticas utilizadas nas refinarias da empresa Sigma sugerindo adaptações e melhorias nos processos da Transferência e Estocagem. A pesquisa apresenta a construção do Modelo de Referência de alto desempenho para a Transferência e Estocagem bem como sua implementação para a empresa em estudo, focando um modelo padronizado que contenha as boas práticas defendidas pela organização e aprovadas por todas as partes interessadas.

\section{Palavras-chave}

Modelo de Referência, Transferência e Estocagem, Petróleo e Refinaria. 


\section{Abstract}

Mello, Juliana; Diallo, Madiagne (Advisor). Reference Model for High Performance Oil Transfer and Stockpiling. Rio de Janeiro, 2008. 118 p. MSc. Dissertation - Departamento de Engenharia Industrial. Pontifícia Universidade Católica do Rio de Janeiro.

This dissertation aims to develop a study of processes modelling and the identification of the best practices held by the oil refineries of Sigma Co. in order to analyze the performance of their activities and suggesting adaptations and improvements in Oil Transfer and Stockpiling processes. This work presents the high performance reference model construction for Oil Transfer and Stockpiling and its implementation for use in the case studied company, focusing a standard model which contains the whole practices supported by the organization and the sponsors departments.

\section{Keywords}

Reference Model; Transfer and Stockpiling; Oil and Refinery. 


\section{Sumário}

1 Introdução .............................................................................. 16

1.1 Contexto Científico e Tecnológico .......................................................... 16

1.2 Objetivos e Motivação ................................................................. 17

1.3 Posicionamento destes modelos na literatura .................................... 18

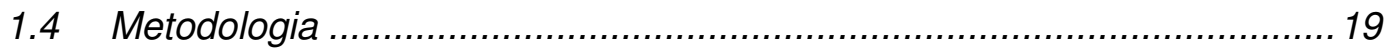

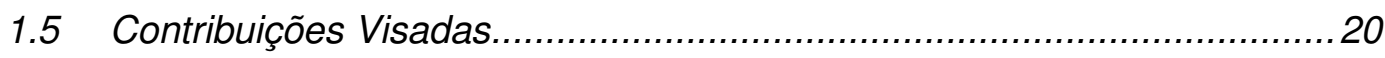

1.6 Descrição e Estrutura da Dissertação ...................................................20

2 Fundamentação Teórica...........................................................................22

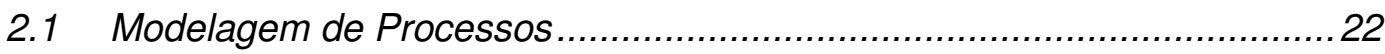

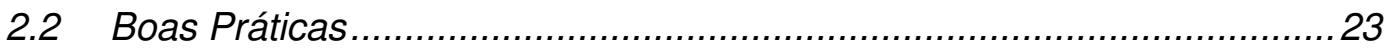

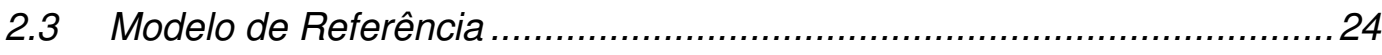

3 Método de Construção do Modelo de Referência.....................................2 27

3.1 Estrutura da Construção do Modelo de Referência .................................28

3.1.1 Workshop Gerencial de Visão da Transferência e Estocagem ...............28

3.1.2 Workshop de Interfaces da Transferência e Estocagem ......................29

3.1.3 Modelagem de Processos na REDUC ..........................................29

3.1.4 Realização de Entrevistas nas Refinarias da empresa SIGMA ............. 30

3.1.5 Análise dos Produtos oferecidos por Fornecedores ............................ 31

3.1.6 Workshop de Discussão das Melhores Práticas na Transferência e

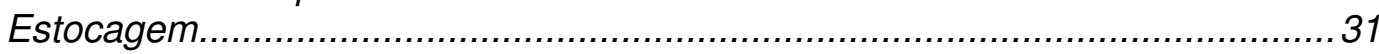

3.1.7 Validação dos Processos Internos da Transferência e Estocagem com a Transferência e Estocagem - Tecnologia de Refino - Sede.............................. 32

3.1.8 Validação dos Processos de Interface junto à Sede ........................... 33

3.1.9 Apresentação do Modelo de Referência e Método EVTE Completo para os gerentes da Transferência e Estocagem ................................................... 33

3.1.10 Documentação do Modelo de Referência da Transferência e Estocagem......

4 Estrutura do Modelo de Referência para Transferência e Estocagem de Alto Desempenho 35

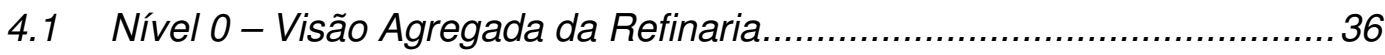

4.2 Nível I - Visão Detalhada da Refinaria ................................................. 36

4.3 Nível II - Visão Agregada dos Setores da Refinaria.............................. 37

4.4 Nível III - Visão Detalhada da Transferência e Estocagem

4.5 Nível IV - Visão Detalhada dos Processos da Transferência

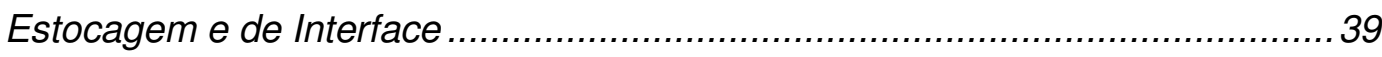

5 Análise do Modelo de Referência ............................................................. 41 
5.1 Nível 0 - Visão Agregada da Refinaria.............................................. 41

5.2 Nível I - Visão Detalhada da Refinaria............................................... 42

5.3 Nível II - Macroprocessos Detalhados da Refinaria ...............................43 43

5.3.1 Nível III - 1. Programação de Produção de Derivados na Refinaria...... 43

5.3.2 Nível III - 2. Gerenciamento do Recebimento de Petróleo e Expedição

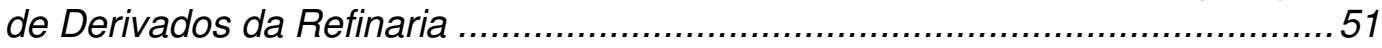

5.3.3 Nível III - 3. Operação da Unidade de Processamento..........................57

5.3.4 Nível III - 4. Vendas e Faturamento da Expedição de Derivados...........58

5.3.5 Nível III - 5. Suporte às Atividades da Refinaria ..................................64 64

5.4 Nível I - Transferência e Estocagem.................................................... 70

5.4.1 Nível II - Visão Detalhada da Transferência e Estocagem.................... 70

5.4.2 Nível III - 1. Programação das Atividades da TE ................................ 71

5.4.3 Nível III - 2. Atividades de Movimentação do Produto ......................... 74

5.4.4 Nível III - 3. Atividades de Preparo de Produto................................... 89

5.4.5 Nível III - 4. Atividades de Preparo de Produto.................................. 95

6 IMPLEMENTAÇÃO DO MODELO DE REFERÊNCIA ........................... 101

7 CONCLUSÃO .......................................................................... 113

8 REFERÊNCIAS BIBLIOGRÁFICAS ................................................ 116 


\section{Lista de Figuras}

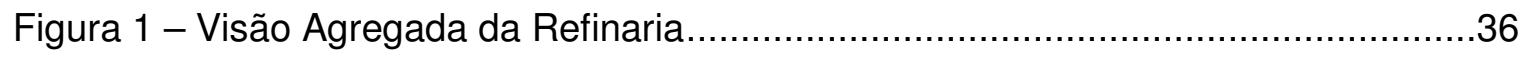

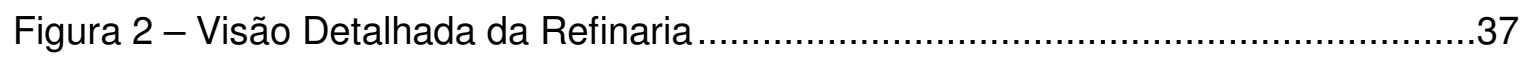

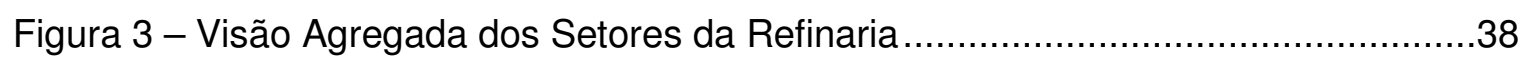

Figura 4 - Visão Detalhada da Transferência e Estocagem .........................................39

Figura 5 - Visão Detalhada dos Processos...............................................................40

Figura 6 - Macroprocesso Agregado da Refinaria .................................................. 41

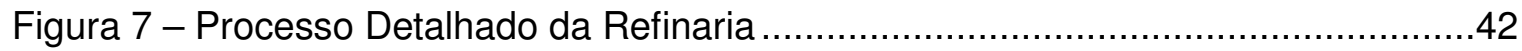

Figura 8 - Macroprocesso da Programação de Produção de Derivados da

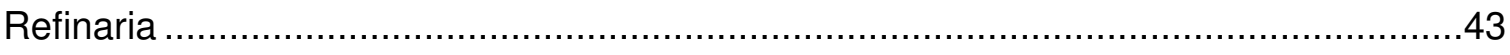

Figura 9 - Macroprocesso do Gerenc. do Receb. de Petróleo e Expedição de

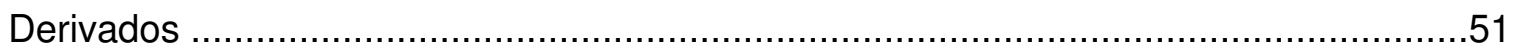

Figura 10 - Macroprocesso da Operação da Unidade de Processamento ......................57

Figura 11 - Macroprocesso de Vendas e Faturamento da Expedição de

Derivados .59

Figura 12 - Macroprocesso do Suporte às Atividades da Refinarias ............................65

Figura 13 - Macroprocesso Detalhado da Transferência Estocagem...........................71

Figura 14 - Macroprocesso da Programação das Atividades da TE .............................72

Figura 15 - Macroprocesso de Atividades de Movimentação do Produto........................74

Figura 16 - Macroprocesso das Atividades de Preparo de Produto ................................89

Figura 17 - Macroprocesso de Suporte às Atividades da Transferência e

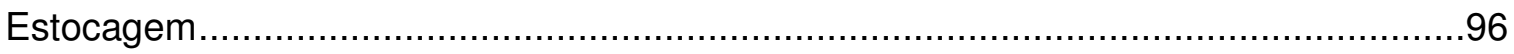

Figura 18 - Tela referente aos controles do menu principal ....................................102

Figura 19 - Tela referente à seção de ajuda ............................................................103

Figura 20 - Tela para selecionar o processo a ser analisado.....................................104

Figura 21 - Tela para visualizar o detalhamento da função .......................................105

Figura 22 - Tela para visualizar apoio à leitura do modelo.......................................106

Figura 23 - Tela para visualizar Boas Práticas de Gestão da TE ..............................107

Figura 24 - Tela para visualizar Boas Práticas de Automação da TE .........................108

Figura 25 - Tela para visualizar Boa Prática de Sistemas.........................................109

Figura 26 - Tela para visualizar Boa Prática de Gestão dos processos de Interface.

Figura 27 - Tela para selecionar qual Boa Prática da TE será analisada.

Figura 28 - Tela para visualizar os detalhes associados às Boas Práticas da TE........112

Figura 29 - Trajetória do Desenvolvimento da TE.....................................................114 


\section{Lista de Tabelas}

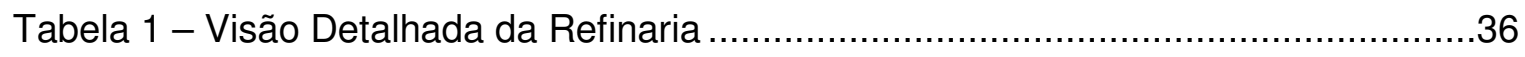

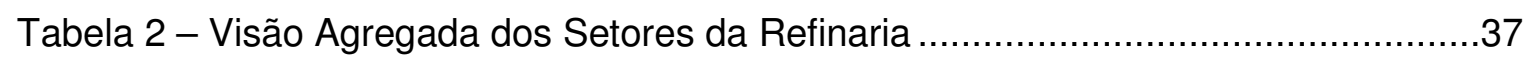

Tabela 3 - Visão Detalhada da Transferência e Estocagem .........................................38

Tabela 4 - Visão detalhada dos Processos da TE e de suas Interfaces ........................39

Tabela 5 - Programação de Produção de Derivados da Refinaria ..................................44

Tabela 6 - Nível IV - 1.01 Elaboração do Plano de Produção ......................................44

Tabela 7 - Nível IV - 1.02 Reunião Diária de Produção - Acompanhamento da

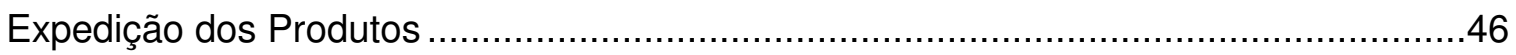

Tabela 8 - Nível IV - 1.03 Reunião Quinzenal do Planejamento Colaborativo ................47

Tabela 9 - Nível IV - 1.04 Programação das Unidades de Processo e da

Transferência e Estocagem .................................................................................48

Tabela 10 - Nível IV - 1.05 Controle da Programação das Unidades de Processo

e da Transferência e Estocagem

Tabela 11 - Gerenciamento do Recebimento de Petróleo e Expedição de

Derivados da Refinaria .52

Tabela 12 - Nível IV - 2.01 Controle de Agendamento para Expedição Dutoviária .......52

Tabela 13 - Nível IV - 2.02 Controle de Agendamento para Expedição Rodoviária ......53

Tabela 14 - Nível IV - 2.03 Programação para Expedição Rodoviária para

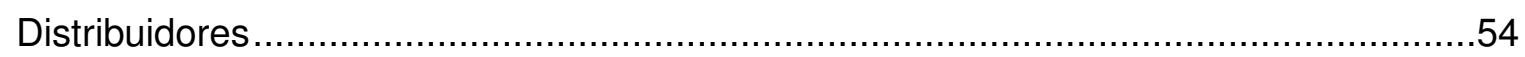

Tabela 15 - Nível IV - 2.04 Controle do Recebimento de Petróleo .................................55

Tabela 16 - Nível IV - 2.05 Controle de Expedição de Derivados para

Distribuidores... .56

Tabela 17 - Operação da Unidade de Processamento ..............................................57

Tabela 18 - Nível IV - 3.01 Operação das Unidades de Processo ...............................58

Tabela 19 - Vendas e Faturamento da Expedição de Derivados ...................................60

Tabela 20 - Nível IV - 4.01 Planejamento de Vendas...............................................60

Tabela 21 - Nível IV - 4.02 Controle da Comercial Diário via Dutoviário e

Rodoviário

Tabela 22 - Nível IV - 4.03 Controle da Comercial Mensal via Dutoviário e Rodoviário

Tabela 23 - Nível IV - 4.04 Controle da Balança .......................................................63

Tabela 24 - Nível IV - 4.05 Faturamento Diário ........................................................64

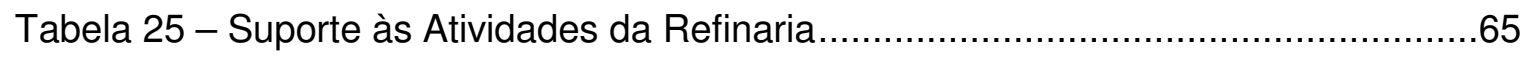

Tabela 26 - Nível IV - 5.01 Ocorrência de Anormalidades .......................................66

Tabela 27 - Nível IV - 5.02 Gestão da Manutenção ...............................................66 
Tabela 28 - Nível IV - 5.03 Tratamento de Solicitação de Ordem de Trabalho. 67

Tabela 29 - Nível IV - 5.04 Elaboração de Análise de Risco ........................................68

Tabela 30 - Nível IV - 5.05 Análise de Amostra de Laboratório....................................68

Tabela 31 - Nível IV - 5.06 Sala de Cálculo ...........................................................69

Tabela 32 - Programação das Atividades da Transferência e Estocagem ......................72

Tabela 33 - Nível IV - 1.01 Programação das Atividades do Turno ................................73

Tabela 34 - Atividades de Movimentação do Produto.....................................................75

Tabela 35 - Nível IV - 2.01 Gestão das Atividades de Movimentação do Turno..............75

Tabela 36 - Nível IV - 2.02 Recebimento Dutoviário ...................................................76

Tabela 37 - Nível IV - 2.03 Recebimento Rodoviário ..................................................77

Tabela 38 - Nível IV - 2.04 Troca de Tanques no Recebimento da Unidade .................78

Tabela 39 - Nível IV - 2.05 Transferência entre Tanques ..........................................79

Tabela 40 - Nível IV - 2.06 Troca de Tanque para Envio para Unidade .........................79

Tabela 41 - Nível IV - 2.07 Expedição Dutoviária ...................................................... 80

Tabela 42 - Nível IV - 2.08 Expedição Rodoviária ...................................................... 81

Tabela 43 - Nível IV - 2.09 Blending Automático ........................................................ 82

Tabela 44 - Nível IV - 2.10 Execução do Alinhamento............................................... 84

Tabela 45 - Nível IV - 2.11 Partida ou Parada de Bombas .........................................86

Tabela 46 - Nível IV - 2.12 Monitorar Atividades de Movimentação................................87

Tabela 47 - Nível IV - 2.13 Otimizar Atividades de Movimentação ...............................88

Tabela 48 - Atividades de Preparo de Produto ...........................................................90

Tabela 49 - Nível IV - 3.01 Gestão das Atividades de Preparo de Produto do

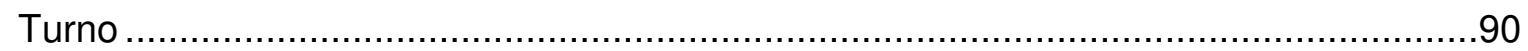

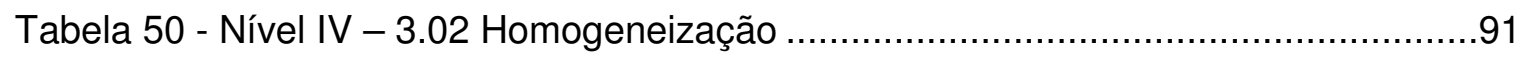

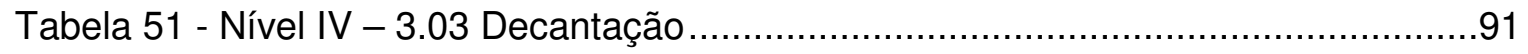

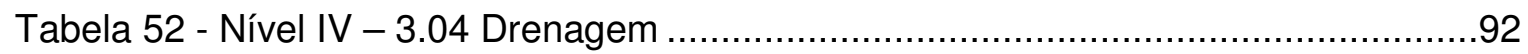

Tabela 53 - Nível IV - 3.05 Coleta de Amostra do Produto .........................................92

Tabela 54 - Nível IV - 3.06 Controle da Atividade de Preparo de Produto ......................93

Tabela 55 - Nível IV - 3.07 Controle de Qualidade dos Produtos.................................93

Tabela 56 - Nível IV - 3.08 Correção de Especificação de Produtos Não

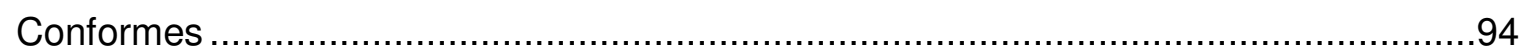

Tabela 57 - Suporte às Atividades da Transferência e Estocagem................................97

Tabela 58 - Nível IV - 4.01 Vistoria de Área........................................................97

Tabela 59 - Nível IV - Liberação dos Equipamentos no Parque TE ..............................98

Tabela 60 - Nível IV - 4.03 Recolocação dos Equipamentos no Parque TE ...................99

Tabela 61 - Nível IV - 4.04 Revisão dos Procedimentos e Práticas da TE......................99 


\section{Lista de Abreviaturas e Símbolos}

CC-Duto Sistema de agendamento de recebimento e expedição dutoviária

CC-Caminhão Sistema de agendamento de recebimento e expedição rodoviária

EMED Emissor de Faturamento da Movimentação

EVTE Estudo de Viabilidade Técnica e Econômica

OT Otimização

PDA Personal Digital Assistant

PIMS Process Information Management System

PLANAB Plano Anual de Abastecimento

RECAP Refinaria de Capuava

REDUC Refinaria de Duque de Caxias

REFAP Refinaria Alberto Pasqualini 
REMAN Refinaria Isaac Sabbá

REPAR Refinaria Presidente Getúlio Vargas

REPLAN Refinaria de Paulínia

REVAP Refinaria Henrique Lage

RPBC Refinaria Presidente Bernardes - Cubatão

SCC Supply Chain Council

SCOR Supply Chain Operations Reference Models

SDCD Sistema Digital de Controle Distribuído

SISPE Sistema de Performance da Transferência e Estocagem

TE Transferência e Estocagem

UP $\quad$ Unidade de Processamento 